\title{
To be a TikToker in COVID-19 Era: An Experience of Social Influence
}

\author{
Rossella lodice ${ }^{1 *}$ \\ (D) 0000-0002-4759-3267
}

\section{Concetta Papapicco ${ }^{1}$}

(D) 0000-0003-3240-8740 SC 00000000000 AAG-1834-2019

1 University of Bari "Aldo Moro", ITALY

*Corresponding author: r.iodice2@studenti.uniba.it

Citation: Iodice, R., \& Papapicco, C. (2021). To be a TikToker in COVID-19 Era: An Experience of Social Influence. Online Journal of Communication and Media Technologies, 11(1), e202103. https://doi.org/10.30935/ojcmt/9615

\section{ARTICLE INFO}

Received: 24 Oct 2020

Accepted: 22 Dec 2020

\begin{abstract}
Social networks are able to interface technological innovation and communication with sociality, thus redesigning the forms, practices, purposes of access and its relationship with everyday life, especially at a time when technology becomes the only way to feel part of a social group, like the COVID-19 period. The general objective of the research is to understand the experience of fruition of the social network Tiktok in the sample of participants, heterogeneous and representative of the reference population. The aim is to assess how the user or the entire social group lives the experience of the social because it is assumed that the result of the above experience can become a new form of social influence. The study aims to answer the following research questions: 1 . How do people experience this new social media? 2. Is fun the new form of social influence? The methodology used is quantum-qualitative. It is, in fact, proposed first a Netnographic Analysis of observation and description of the social, and then use an automatic Content Analysis supported by Diatextual Analysis (the qualitative part). The results allow to theorize a different form of social influence, or "fun", which becomes characteristic of the social network and the period.
\end{abstract}

Keywords: Tik Tok social network, virtual group, social influence, quanti-qualitative methodology

\section{INTRODUCTION}

The Digital Revolution has led to great transformations in today's society, generating increasingly sophisticated interaction tools. Digital natives (Prensky, 2001), in fact, are generations able to use new media intuitively. At the same time, new media creates new affordance for digital natives (Gibson, 1979), such as creating virtual groups or generating content. As for the generation of multimedia content, this opportunity is evident starting from the Social Network generation (Riva, 2014), in which, even non-expert users, become creators of posts, images, memes or videos. The content created, then, can be followed, shared, commented or imitated by other users, generating, in this way, a virtual social interaction. Therefore, interactions allow the spontaneous creation of social groups within these virtual environments.

As with offline groups, online ones are not just aggregates of people, but are made up of users who pursue common goals and are committed to their achievement. Online groups are created spontaneously when members focus on their activity, negotiating meanings that affect their interests and their entire existence in an inter-subjective space. One of the significantly influential spaces is Tiktok, above all in the particular moment, that of the lockdown following the COVID-19. Indeed, since the beginning of January 2020, the spread of new coronavirus (SARSCoV-2) emerged in Wuhan in China at the end of 2019 and that is still spreading mainly in a vast region since the epicentre, despite the unprecedented efforts made by China to contain it.

Copyright (c) 2021 by authors; licensee OJCMT. This article is an open access article distributed under the terms and conditions of the Creative Commons Attribution License (http://creativecommons.org/licenses/by/4.0/). 
Italy was the first European nation to apply rescrictive politics, as social distancing and lockdown (Papapicco, 2020, 3). What influence, then, do social networks have in this period of distance? Tiktok, in line with other trendy social networks, presents itself as a social of creativity, a social of action, whose main function is to inspire the creativity of the other. The objective of the research, in fact, has inserted into the context of the social use and revealed a knowledge in the psychological field: 1 . How do people experience this new social media? 2. Is fun the new form of social influence? Through the Snowball technique of recruitment, 30 participants took part in an empirical survey, suitable for obtaining comparable results. From survey, analysed through quanti-qualitative methodologies, it emerges that the experience of social use is readily correlated with a self-directed fun compared to a joy of sharing. The social experience was evaluated through questions such as "How do you feel emotionally about receiving credit from followers?", necessary for the collection of valuable information symbol of semi-objectivity in the experience of social network fruition.

\section{FROM ONLINE SOCIAL GROUPS TO CREATIVE AND IMITATIVE CONTENT CREATION}

Groups are a complex reality, with dynamic and specific phenomena that characterize them, that make them intelligible and predictable. The continuity or discontinuity of group life is determined by elements that act as a "centripetal call" causing the group to remain united over time, although the group as individuals experience continuous changes and, by "centrifugal forces" that contrast group stability by generating a "resonance" within the group that would disrupt cohesion and foster the change of the group micro-part or member.

After having become aware of opinions/beliefs shared by other people, changing opinion or embracing a new belief is related to the "social" process, fundamental to social relations and interpersonal influence. The social influence is the behaviour by means individuals tend to conform to the ideas supported by the majority, without being asked explicitly (Asch, 1955).

Some scholars attribute to this phenomenon socially disapproved behaviours: as shown by the studies conducted by Stanley Milgram (1974) on destructive obedience. The studies about obedience aimed at excluding and harming other people, as well as the result of personal needs or orientations or forms of moral disengagement may depend on processes of social influence that occur in the context of interpersonal relationships.

A significant indicator of openness to the new virtual world allows to identify, in the singular sphere of social networks, an anti-social conduct that allows to highlight a population of competent users with respect to the media sphere, to their languages, to the plurality of media offers and to their ability response at different needs.

Social networks are able to interface technological innovation and communication with sociality, thus redesigning the forms, practices, purposes of access and its relationship with everyday life, in its multiple dimensions. The ramification of the social process of interpersonal influence is deepened on the two sides represented by the real and the virtual world. The way in which humans behave in online environments discusses generalizations on the way in which environmental and personal determinants influence behaviour in the different contexts that use technology.

The term 'social presence' refers to the degree to which an individual is perceived as a "real person"; the mediation of technology influences this perception and human interactions, especially in differentiating the ability to transmit non-verbal information. A warm and friendly interaction will be perceived by interlocutors who use a medium able to convey a greater number of verbal clues. In face-to-face encounters, multiple nonverbal clues are combined, in real time, with spoken words. In computer-mediated contexts, based primarily on speech, users adapt to the context using any available means to convey emotions. Despite the frequent limitations of the world of the Web, as reported by the most popular media, it is recognizable, within the same environment, the birth and development of relationships to an intense extent. These communications are able to support "hyper-personal" communications, with a high degree of intimacy. 
The immeasurable accessibility, that appears in the world of social with their unique peculiarities aiming the amount of sources of knowledge, leads the online person to identify with an online group; to comply with its rules and to overestimate the similarity between its members. The following paragraphs interpret the phenomenon of media influence by outlining the interaction between the digital native and the social network privileged in the Tiktok social network. The "digital native" (Prensky, 2001), through the new technologies that act as a support for his expressive imagination, creates multimedia content in an autonomous and collaborative way. The aspect of content creation amplifies the creative potential of the individual and reflects the conscious choice to produce original content among peers. The close proximity between the user and the social network promotes the birth of intra and inter-relational abilities, such as interactivity that, in the individual dimension, can be translated into the term "sense of initiative". The person's ability to translate ideas into action is encouraged by the use of the social network through which 'tiktokers' share their creativity.

\section{PARTICIPANTS}

The respondents' group to the empirical study were initially recruited among young people. Through the heterogeneity of the 30 participants, the evaluation of the experience of using the social was built in compliance with objectivity, validity and sensitivity.

The recruitment of participants took place by means of a non probabilistic sampling mode: Snowball sampling starts from a number of sampling nodes, having the required characteristics (i.e. to be adolescents) and each of the selected cases indicates the contact persons having the same characteristics. The avalanche technique is constructed in a way as to guide a spontaneous detection that does not foresee hypotheses about the research phenomenon. The so-called "word of mouth" was a sign of lightness in the desire of teenagers to answer questions regarding their experience of using social media. The survey was carried out through the Google Modules platform and included 2 multiple choice questions, a question with short answer text and 5 open questions, from which immediately emerged the purpose of Tiktok use. The social experience was evaluated through open questions, such as "How do you feel emotionally about receiving credit from followers?", representative of the tiktoker's experience, as shown in Figure 1.

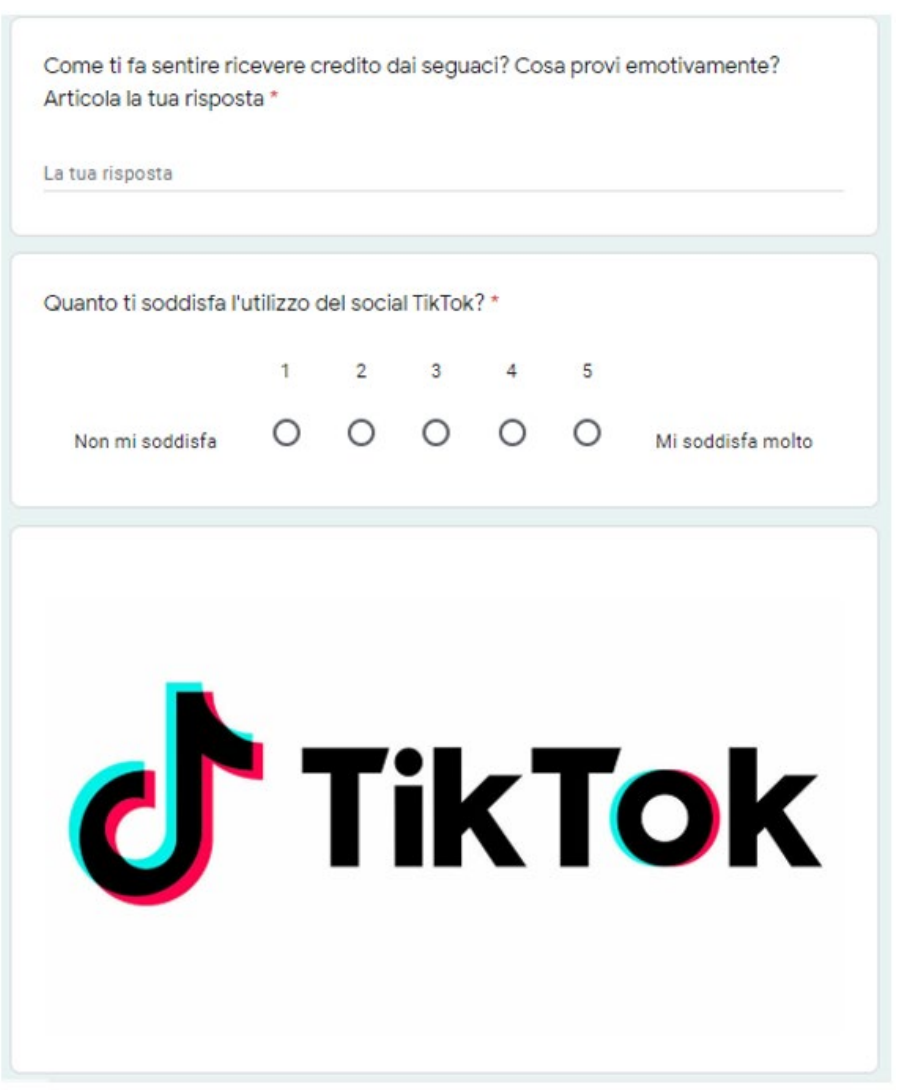

Figure 1. Survey Layout 
The survey found a sample of heterogeneous respondents characterized by $63.3 \%$ from users using female Tiktok and $36.7 \%$ male gender. The procedure has also registered among participants a $20 \%$ between 12 and 16 years, a 53.3\% between 17 and 21 years, a $16.7 \%$ between 22 and 26 years and a $10 \%$ between 27 and 31 years. The majority of teenagers also stated that they were $76.7 \%$ satisfied with using Tiktok to unleash their creativity.

\section{HYPHOTESIS, OBJECTIVES AND METHODOLOGY}

The general research objective is to understand the experience of the social network fruition, in the specific case of Tiktok for the sample of participants, heterogeneous and representative of the reference population. The aim is to assess how the user or the entire social group lives the experience of the social networks because it is assumed that in TikTok experience there might be a new form of social influence.

The study aims to answer to the following research questions:

1. How do people experience this new social media?

2. Is there a new form of social influence?

Among the specific objectives, the research aims to reflect on the uniqueness of Tiktok's experience compared to other social networks.

The methodology used was the quanti-qualitative one. For the qualitative part, a Netnographic analysis (Kozinets, 2016) was carried out, useful to describe the social and subsequently through a Content Analysis and a Diatextual analysis (Papapicco \& Mininni, 2019). Netnographic analysis provides information on the meanings and behavioural patterns of online communities; the guidelines of the Tiktok online environment have outlined a platform that determines different experiences of the experience: the use of social as a showcase to appear, as dictated by fashion, stands in the way of an experience of fun linked to the self and its satisfaction. The intrinsic openness of ethnography promotes a variation related to the fun construct, which is measured on the basis of user habits and found to be less suitable for sharing experience. The sharing of the experience, prevalent compared to the publication of the funny video (real purpose of the social) is a procedure studied, instead, through the analysis of the content. The support of the Sketch Engine platform has contextualized verbal expressions within their respective periods making relevant information seemingly obsolete. The description of the experiences has adhered to a paradigmatic notion of the experimental phenomenon. From the frequency of their expressions and focus on their position within the logical proposition, a positive correlation between the experience of using the social and the influence/dependence on the entertainment generated has emerged. The Diatextual Analysis, instead, through the "SAM MODEL" (Mininni, 2007) has developed the sense-making of the answers object of the experimentation; whose markers of agentivity and affectivity were the criteria by which the theorization of a correlation between the experience of social use and the experience of fun was arrived at.

\section{Analysis procedures: Content Analysis and Diatextual Analysis}

The study of internal speech devices allows the evaluation of fluid interaction because coordinated between the interlocutors. The intersubjective understanding of the user-social group and user-social network relationship takes into account the development of the contents expressed/requested. The social construction of the meaning produced by a speech through rhetorical or content aspects assert a common linguistic "repertoire" (Edley, 2001) in the oral and written form of adolescents. The Content Analysis (Mininni, 2013) is a tool for the study of codes through which the meanings and rules are recognized, the response that varies depending on the interlocutor and the function of the speech specific fulfills and located interational purposes.

The interpretation techniques adopted by the Sketch Engine (Kilgarriff et al., 2004) software have given meaning to the frequency of verbs and the position of "verbs in context", whose consequentiality or spatial inconsistency have been indicators of familiar conceptualizations to the research. The fun experience is expressed through the representation of graphs that report the relationship between the tiktokers and their experience with the social Tiktok. 
Diatextual Analysis (Mininni, 2013), falling into a more holistic dimension, uses procedures capable of capturing the meaning of a communicative event identifiable in the experience of a new form of social influence. The configuration of the SAM model (Mininni, 2013, p. 43), along the dimensions of Subjectivity, Argumentativity and Mode, organize a coherent profile, that produce sense. The meaning constituted by "argumentative contexts" classifies the present link between the structuring words of a speech and the predicates, which acquire a sense if inserted in the argumentative frame of reference. For example, "Reaching a high number of video views" is an expression that can be traced back to the sense attributed by the teenager only after the acquisition of common expressions, a symbol of phenomena that characterize the age group of reference.

\section{RESULTS}

\section{Netnographic Analysis}

The Social Network, at the center of the procedure of social research, has placed itself as a means for Netnographic Analysis, as an important evaluator to better orient the sensitivity of research. The implementation of a theorization aims to generate social spaces and territorial areas whose explanation describes and enhances the participation of subjects in the online world. The objective of the Netnographic Analysis is to probe and analyze the perception of users in the fruition of the social network, connecting the communicative codes and the meanings attributed to the emotions aroused.

In Tiktok social network, the thoughts expressed by the users are divided into macrocategories of analysis and classified according to the perception that the users express. The qualitative dimension pertaining to the users' reactions with respect to the present insight is substantiated in the interpretative analysis of the answers to which the participants have been submitted. Analyzing a "trend" construct of the range in teenagers' group has provided useful information about the expressive codes inserted in the social context. For instance, "Receiving credit makes me feel satisfied and recognized at a social level". This purpose is the key that disrupts the sensitive points of communication and ranks as the starting point for designing a prototypical and representative analysis of the tiktoker. The user, after registering to the platform, has 15 seconds of video to make a lip sync on the most popular songs and give vent to his skills as a performer during the staging of a dance or a video in which the protagonist portrays his expression (editable through predefined filters) during a singing scene. This is followed by the access to the Tiktok social network, characterized by the exchange of comments and the selection of profiles to add. By turning on the camera of the device, through the Tiktok application, it can be chosen the song to be filmed and start recording, which can be slowed or speeded up at the wish of the user who will decide whether to publish or archive the video.

\section{Quantitative and Qualitative Results}

The quanti-qualitative research has placed at the center of the investigation the degree of influence of the Tiktok social network and its correlation with the ability to generate dependency. Operationalizing the construct has meant articulating the degree of dependence in two variables, qualitatively measurable, in order to verify whether it is the social, as well as object of analysis, to generate dependency or what is possible to achieve through the social.

The research hypothesis, focused on the feeling of "wanting to have fun", has shed light on the dynamics of interaction between the influence from Tiktok and the addiction to fun, accessible through the social network mentioned.

For the purpose of the survey, it was useful to decline the objective of the research, contextualize it and separate the variables related to the dependency construct.

In order to reach the research objective, an analysis has been carried out on different age groups, which are individually responsible for behaviours characterizing the "age" variable involved.

To contextualize the construct it was necessary to look at the unpleasant situation that involved the entire globe: the Coronavirus pandemic that has suddenly and profoundly altered the habits of individuals, making it interesting to evaluate the social experience in youth and adults in the pre-covid and post-covid experience. 
To separate the variables related to the dependency construct, instead, was analyzed the proportionality of the degree of dependency on social or entertainment that can be reached through the social.

The survey was completed by a satisfactory number of people and allowed the analysis of the results to reveal facets to which the research hypothesis was not oriented but at the same time, complete the social and relational background of Tiktok social network.

Tiktok has attracted the attention of adolescents and adults with prevalence of teenagers; the reference sample of research and representative of the range of subjects belonging to the social sphere, placed emphasis on divergence in habits in relation to age of use, as shown in Graph 1.

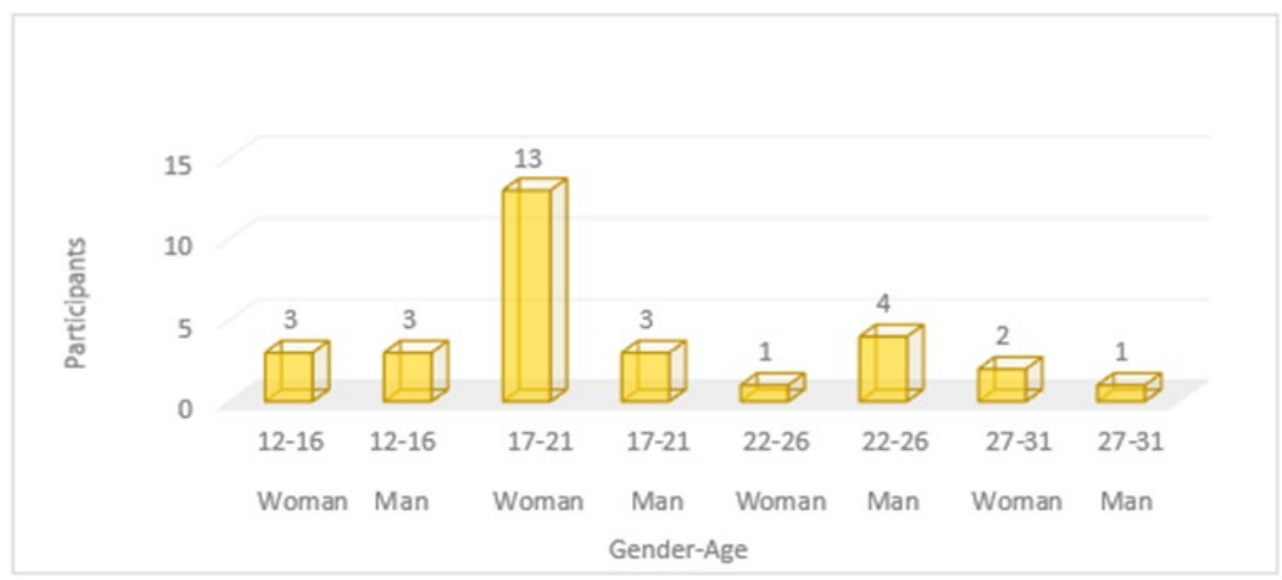

\section{Graph 1. Relation Gender and Age of respondents}

As it can see from the Graph 1, from the crossing of the age variable with the gender variable, it is possible to recognize a prevalence of use in the 17-21 age group. As evidence of this in the literature (ISTAT, 2018), the survey confirms a higher frequency of use among teenagers of female gender. For degree of classification but not for importance, the 22-26 age group is placed as second with an evident dichotomy linked to the gender variable. Although women may be defined as predominant in terms of participation in social network, in the first case, in the 17-21 range, the assumed data supported by reliable statistical data (ISTAT, 2018) are confirmed, while in the second case it is easy to identify a dissonance, linked to an increase, related to the 2226 age group, of the male gender in contrast to the statistics available (ISTAT, 2018).

Analyzing the online behaviours of the research participants appeared immediately known as focus on fun, oriented towards the self or towards others. Tiktok's experience has proven to generate self-directed and non-direct fun, fun is not created to be exchanged or shared. The self-contained fun construct has been split into two sections: the youth band and the adult band, not prevalent and therefore not predictable social behaviour in full.

A self-directed sense of fun in the adult group shows itself as a diversion because linked to the present moment, of loss of individual freedom and contact with the most dear affections.

It is easy to define behaviour as a diversion when the action of the young people group moves in another direction. The data obtained, however, show how easily it is possible to fall into the error of a diversification of behaviours and how close the behaviours of the two groups are. Creating fun is a primitive matter of need, a normal condition of the human being (Borghi \& lachini, 2004): young people, in the same way as adults, create fun to satisfy their sense of fulfillment and resulting in a normal condition in the group of teenagers; it is possible to recognize a heterodirect sense among adults, albeit in lesser quantity, as a misunderstanding of the purpose of the social and as a showcase for the appearance.

For the purpose of a wider and more complete analysis, the interview, given in written form to those who have decided to take part in the research, has requested as additional information but necessary the association of the title of study, as shown in Graph 2. 


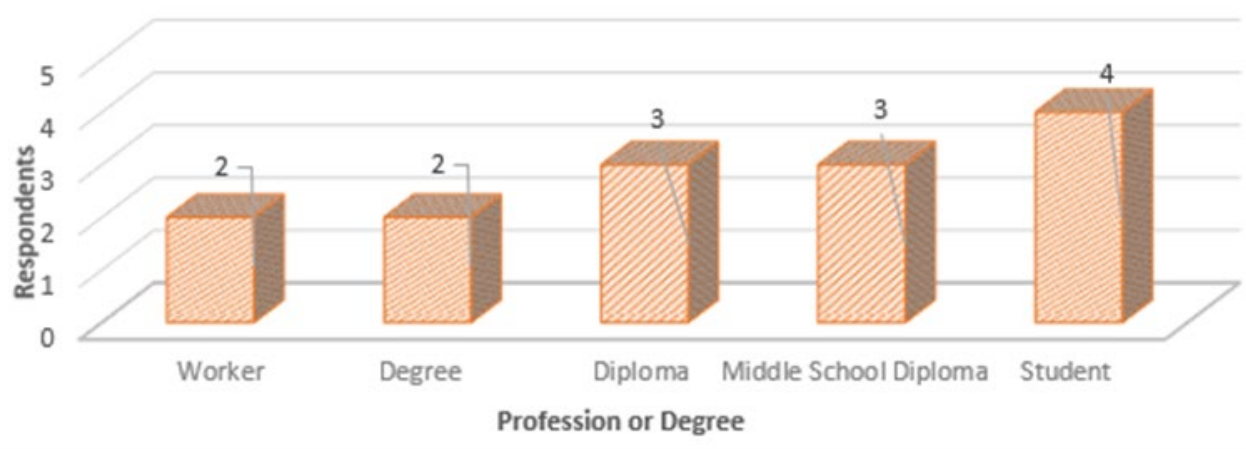

Graph 2. Occupation or qualification of respondents

Among the participants, a predominance of students emerged, confirming what supported by the hypotheses. Those who use the Tiktok social network categorize themselves as possessing the "college degrees" as a qualification and practicing "student" as a profession. The percentage of workers is smaller, evidently due to temporal issues and lack of interest and graduates, probably users of other social networks able to attract more attention. The not insignificant percentage of those who have as a single recognition of education the "Middle school License" opens new horizons towards a more in-depth analysis and aimed at understanding the motivations that push them to the use of social; a small deduction would lead to associate a low level of education with a high level of use of socials for reasons of lack of interest in traditional information channels.

Tiktokers, in fact, use social media not to document personal knowledge but to satisfy their need for fun and creativity. The fulfillment of a desire is recognized, in the psychological literature (Maslow, 1954), as a need for self-realization, as a natural need of the human being for inner growth. The participants themselves manifest a clear realization of their creativity through the use of social media, as shown in Graph 3.

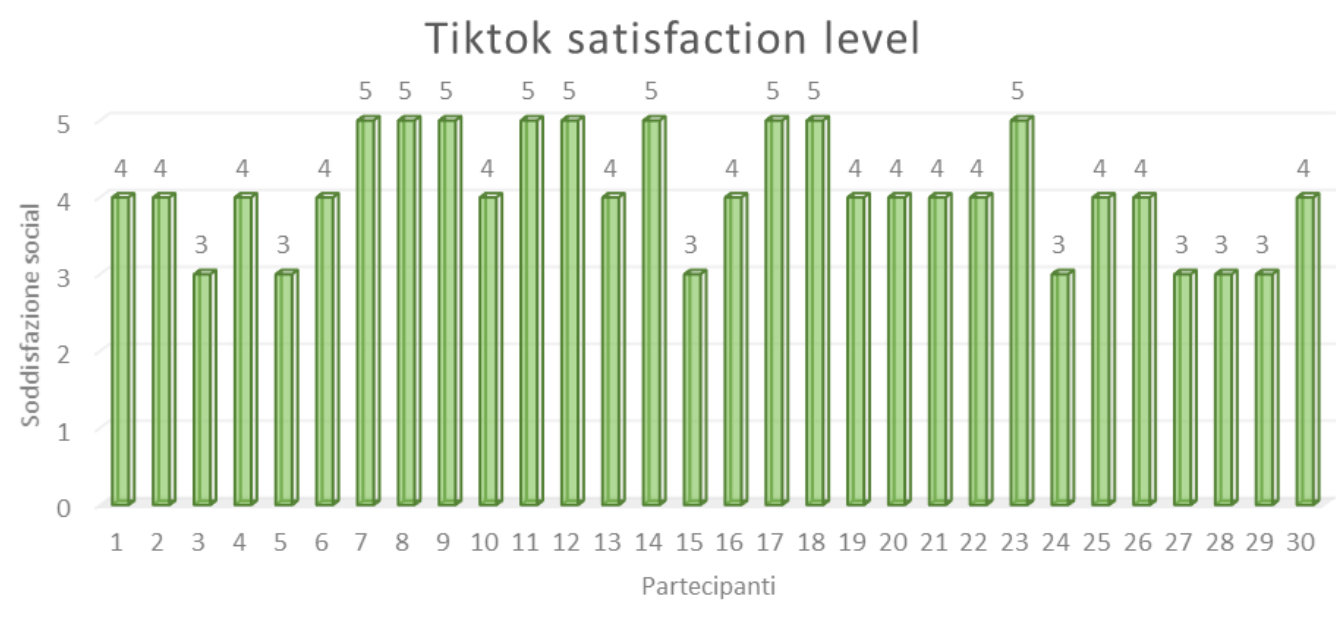

Graph 3. Tiktok satisfaction level

The Tiktok social network is a social action, i.e. a social that inspires the creativity of users but especially experienced as social fun: the prevalence of fun and creativity leaves little room for imitation of an idol, very frequent, however, on other social networks such as Instagram or Facebook.

It happens because creating (a video) determines two different experiences: "Recording" and "Doing". These are also verbs that define the experience of a self-directed fun, which is opposed by a small number of users who attribute to the fun a heterodirect sense. The concept of social use to appear is common among female participants, who declare to find the social fun for the irony and sarcasm that manages to graft, as in the following examples: 
E.g. 1 "It allows us to be who we want to be, to confront ourselves with others and our chameleon ability to put ourselves in the shoes of others";

E.g. 2: "The beauty of the app is just that, the fact that everyone tries to entertain as he likes".

"I'm thrilled that people try their best to make each other laugh" and the irony of the single contrasts with a marginal presence of teenagers who "enhances" the creation of videos with friends:

E.g. 3 "I'm excited to create videos all together".

The category of "visibility" or "appearance" has placed a small number of answers, corresponding to the value of the attitude of exposure to the other; the low frequency of imitation of idols, emerged from the sample, is a mechanism of "débrayage" which moves values such as "External Involvement" and "Emotional Detachment" away from the average.

The fun, translatable on the social at stake, singing and "chatter", classifies the social as a useful tool to "Leave the reality behind"; it is the speed and accessibility to the pleasant distraction to arouse feelings of well-being:

E.g. 3 "It cheers the day".

Exchanging irony and fun with nice expressions is a real discovery for teenagers, which given the criticality of the situation Coronavirus and physiological change of their age, in contradiction with compliance and "fashion". They discover and begin to prefer interaction with the relative.

The absence of interpersonal relationships, due to the unpleasant situation COVID-19, is, in fact, a possibility to recognize Tiktok as a social familiarity, as demonstrated by the recurrence of association of the verb "Do" and the noun "Relatives". Diatextual analysis presents a concept of fun that is not shared or "brought to the outside" but "brought to the inside", towards one's own family.

The friend plays a crucial role in everyday life but not in moments of psychological default such as the one marked by the contemporary pandemic of COVID-19.

The sharing with the relative and the rediscovery of a common pleasure, often not valued during adolescence, is accompanied by the enhancement of their individuality to which Tiktok offers great importance.

Through effective para-verbal communication, therefore through facial expressions, the social allows the individual to represent his own individuality, free from the canons and external stereotypes, typical of sharing. A pleasure aimed at being of personal and external satisfaction allows the individual to confront himself and increase his own self-effectiveness thanks to the "chameleon ability to put himself in the shoes of others".

It is used to know but above all for pleasure. The staticity of verbs is an indication of the rejection of vivacity, of shared activism that orients the user's action towards a "show to himself" and a "pleasure to himself". Preferring one's own person to a friend or acquaintance means focusing attention on one's own interiority, towards that "new" never known.

The prevalence of the state verb, as mentioned above, is an index of the static action as opposed to the verb "Do" often correlated with "Feel" and "Satisfaction" as proof of that personal satisfaction that induces, mostly teenagers, to become users of a new social network driven by curiosity towards something new, as shown in Figure 2. 


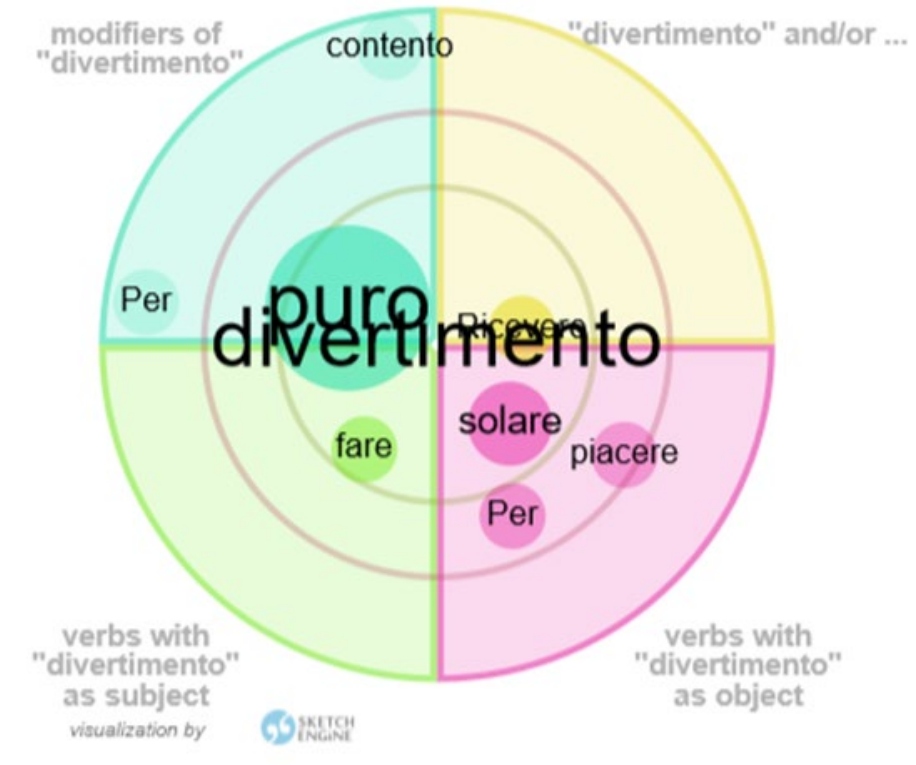

Figure 2. Sketchword on Tiktok users

"Downloading" ('Scaricare') the app and "Becoming" ('Diventare') famous on social networks are the most recurring expressions among the 'diatexts' of the participants: they are actions in progress, whose cyclicality is interposed by fashion that leads the teenager to download the social application not for sharing of video (real purpose of the social) but through the sharing of experiences. The download is different from becoming a tiktoker and this is relevant to the sharing of the experience, fundamental to the eyes of a teenager.

Being linked to satisfaction and pleasure and less to the sharing of experiences makes the social spokesman of an inner need of the individual, a fun that, driven by external factors, is not heterodirect. Forced social isolation has repercussions on psychophysical well-being and beyond, some research (Pfefferbaum, B. e North, CS, 2020) shows how a painful experience such as uncertainty about the future and one's own health, linked to the COVID-19 situation, both generating feelings of anger and phobic evasion behaviour. The cancellation of social contact is referred to as the cause of negative feelings such as demoralization and a sense of isolation from the rest of the world.

In the light of a life marked by a bitter nuance, the dependence construct acquires a different importance at the basis of the identification of a causal link between the user's behaviour and the environment that surrounds him. When closing tightly the user shows imposing a further closing, legitimizing a fun directed only towards himself.

The recurring verb in the interviews administered is "Enthuse", given by a joke or a dance that in 15 seconds is played on social media, as in the following example:

E.g. 4 "The fact that you danced excited me"

For instance, this extract offers a new perspective of fun as excitment, which is born as inter-group and it becomes intrapersonal. New users claim to enter the social world of Tiktok because the trend requires it, because conforming to a single shared reality allows them to feel part of a group.

Download the social app as a joke, for a bet, out of curiosity or circumstance means changing the original focus of the medium, i.e. the preference of fashion in teenagers' group, apparently, compare their performance with that of a video posted. In reality, the true intent of the teenager turns out to have changed course: that is "capture" the video at will and stage the inspiration received, mostly with a relative.

The verb "watching" ('guardare'), in its transitive function, expands towards the object, towards a source of pleasure: the short video becomes a source of joy and carelessness. That's why, the user does not look for fun from the outside, through sharing and exchange with peers, but records sketches of the duration of a clock ticking to suit their own irony, as shown in Figure 3. 


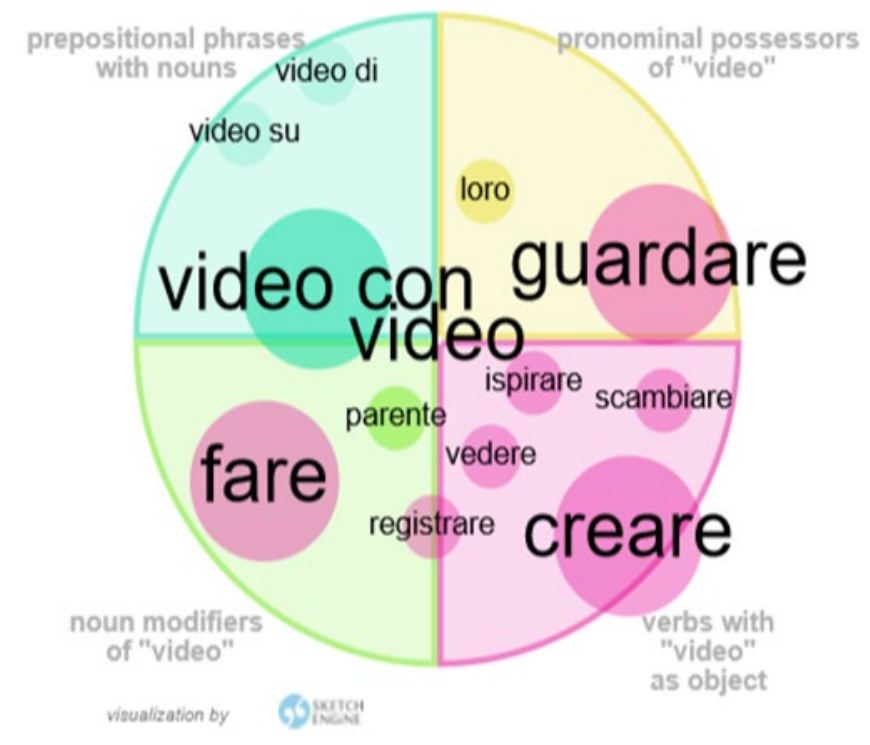

Figure 3. Sketchword TikTok Participant Verbs

Typical of the adolescent temperament, driven by the discovery of what makes the single part of a group, is the application of social results against the social but the experience of social fun.

Analysis using the Sketch Engine software placed emphasis on the habits of Tiktok users that readily appeared as varied but similar in the choice of audience to which to target their successes, as shown in Figure 4.

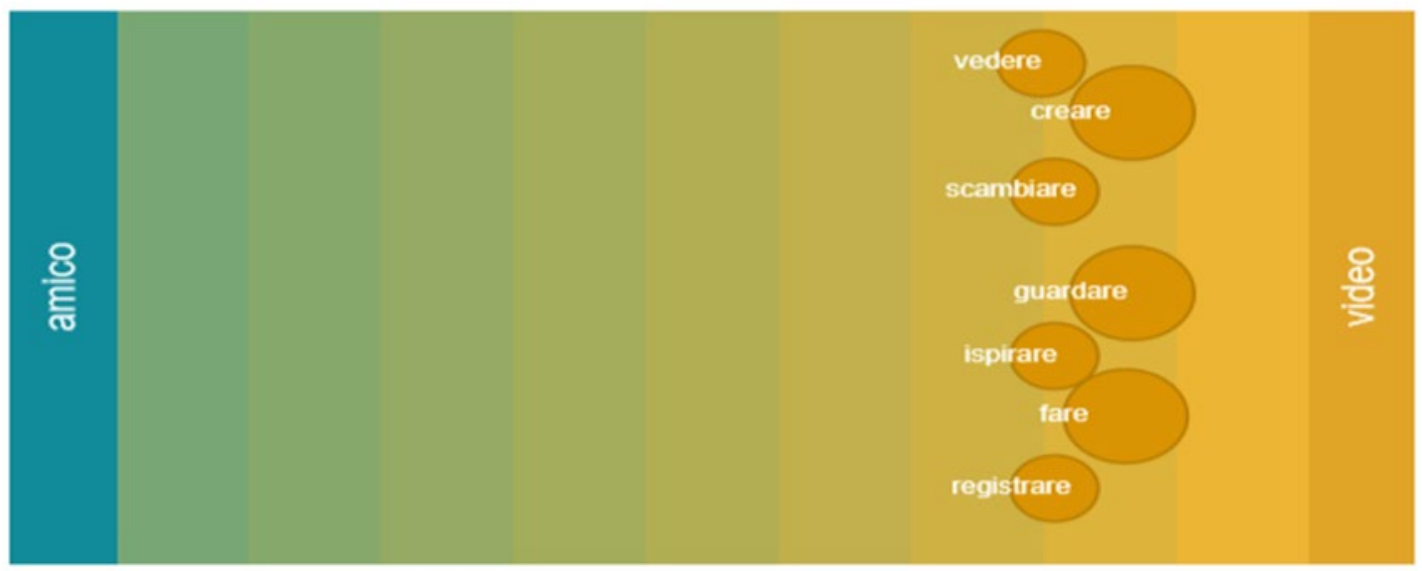

Figure 4. Polarization verbs and words Tiktok

In Tiktok, it is possible to create videos, to download videos, to record videos with the aim to satisfy the interests, for the need of individuals to give voice to their self-esteem; a lack of congruence with the social network defines the medium as a source for personal growth and satisfaction of individual interests.

The Self-realization and the implementation of one's own potentialities are, in fact, the key to enter an inner path of which man cannot do without, effectively undertaken by exploiting one's own intellectual, physical and emotional capacities, essential to become what you want.

The predominance of the verb "To be" ('Essere') emphasizes the no dynamism that distinguishes this "social" medium, defined as "little" social: the user who records video, it does so because it has embarked on a path towards self-realization that requires spontaneity, autonomy, originality and creativity.

Instincts or pleasures take on a positive connotation in Maslow's theory (1954) of self-realization, as they represent an opportunity for growth. For a long time, instincts have been repressed and relegated to the animal kingdom, now they are valued to represent the vital energy of humanity, the heritage of the individual who needs to be rekindled to give light to individual uniqueness. 
Unlike other social platforms, Tiktok is mainly based on entertainment and one of the main features is the ability to express creativity with funny videos, choreographies and lip-sync songs. Making videos of 30-60 seconds combined with music, sound effects and filters, recognizes the real novelty of the platform that offers the opportunity to know their talents. Memorizing videos of other tiktokers and creating their own helps to make the self-efficacy of the individual, mostly teenager, in line with expectations and goals. The ability to imitate and synchronize with the musical beats, in fact, involves experiencing aspects of their own hidden personality and gradually begin to feel comfortable with them.

Recording videos to be stored in the device's gallery and not to be used for sharing shows that the social network is in a small way oriented towards opening the borders with the external environment and although it can be considered not very open to sharing, as demonstrated by the representative examples:

E.g. 4 "Participating in challenges, makes me feel socially accepted",

E.g. 5 "Receiving credit from followers makes me feel satisfied, causes me pleasure".

The placing of the verb "Do" ('Fare') within the statements of the subjects involved in the research emphasizes the meaning attributed to fun by young people, who demonstrate two type of social influence of entertainment:

1. The Self-fun

2. the "Smile of the other".

Through the qualitative analysis it was essential to recognize a rise in the initial attention of the research, oriented towards the verification of dependence on social. Assessing the degree of influence of the social Tiktok to understand its addiction capable of generating in young people and adults opened the way to a new analysis reached concrete results. Using the graphics of the software Sketch Engine the initial question has received more answers than expected: the influence that the social results to be able to generate is not confirmed by the data collected, because to generate dependency is not the creation of the video (real purpose of the social) but the fun that putting the users' talents to test the ability of excitement.

\section{DISCUSSION OF RESULTS AND CONCLUSION}

From the results obtained it emerges that the social network is used for a self-entertainment away from the sharing of experiences: as reported by the literature, the world of social networks is a "small world" where they are all connected with each other. The "globalized" society multiplies the possibilities of contact; at the same time, however, often people can be in different places or between people rather familiar. In a world that makes flexibility and uncertainty its flags, contacts able to activate through the network become one of the first forms of social capital. Online becomes free access to the most intimate daily emotions being every action accessible to a wide audience and shareable by numerous users (Paccagnella \& Agnese, 2016). The fun, therefore, among tiktokers reaches countless environments that amplify the phenomenon itself. It is the reticularity of the web that leads to a theorization of fun as a new form of social influence.

\section{REFERENCES}

Bakshy, E., Rosenn, I., Marlow, C., \& Adamic, L. (2012). The role of social networks in information diffusion. Paper presented at the Proceedings of the 21st international conference on World Wide Web. https://doi.org/10.1145/2187836.2187907

Borghi, A., \& lachini, S. (2004). Scienze della mente.

Edley, N. (2001). Analysing masculinity: Interpretative repertoires, ideological dilemmas and subject positions. Discourse as data: A guide for analysis, 189, 228.

Gibson, PJ (1979, September). L'antenna vivaldi [The vivaldi antenna]. Nel 1979 9th European Microwave Conference.

Hogg, M. A., Vaughan, G. M., \& Arcuri, L. (2016). Psicologia sociale: teorie e applicazioni [Social psychology: theories and applications]. Pearson.

ISTAT- Istituto Nazionale di Statistica (2018). Uso di Internet [Use of the Internet]. 
Kilgarriff, A., Rychly, P., Smrz, P. e Tugwell, D. (2004). Itri-04-08 il motore di schizzo [Itri-04-08 the sketch engine]. Tecnologia dell'informazione-Information technology, 105, 116.

Ma, L., Feng, J., Feng, Z., \& Wang, L. (2019). Research on User Loyalty of Short Video App Based on Perceived ValueTake Tik Tok as an Example. In 2019 16th International Conference on Service Systems and Service Management (ICSSSM) (pp. 1-6). IEEE. https://doi.org/10.1109/ICSSSM.2019.8887751

Mininni, G. (2007). La psycholinguistique à l'oeuvre dans la traduction [Psycholinguistics at work in translation].

Mininni, G. (2013). Psicologia culturale discorsiva [Discursive Cultural Psychology]. Milano: Franco Angeli.

Paccagnella, L., \& Vellar, A. (2016). Vivere online: identità, relazioni, conoscenza [Living online: identity, relationships, knowledge] (pp. 1-202). II Mulino.

Papapicco, C. (2020). Informative Contagion: The Coronavirus (COVID-19) in Italian journalism. Online Journal of Communication and Media Technologies, 10(3), e202014. https://doi.org/10.29333/ojcmt/7938

Pfefferbaum, B. e North, CS (2020). Salute mentale e pandemia di COVID-19 [Mental health and the COVID-19 pandemic]. New England Journal of Medicine, 383, 510-512. https://doi.org/10.1056/NEJMp2008017

Prensky, M. (2001). Nativi digitali, immigrati digitali [Digital natives, digital immigrants]. All'orizzonte.

Priyawan, P., Rusdianti, A., Nurfauziah, A., \& Asmarani, A. (2019). When fans and haters are on the same boat: a deconstructive reading of Bowo and TikTok. Makna (Jurnal Kajian Komunikasi, Bahasa, dan Budaya), 4(1), 128-138. https://doi.org/10.33558/makna.v4i1.1676

Qualizza, G. (2013). Facebook generation: i "nativi digitali" tra linguaggi del consumo, mondi di marca e nuovi media [Facebook generation: the "digital natives" between consumer languages, brand worlds and new media]. EUT Edizioni Università di Trieste-EUT University of Trieste Editions.

Riva, G. (2012). Psicologia dei Nuovi Media [New Media Psychology]. Azione, Presenza, Identità e Relazioni nei Media Digitali e nei Social Media [Action, Presence, Identity and Relationships in Digital Media and Social Media]. Bologna: II Mulino.

Yang, S., Zhao, Y., \& Ma, Y. (2019, July). Analysis of the Reasons and Development of Short Video ApplicationTaking Tik Tok as an Example. In Proceedings of the 2019 9th International Conference on Information and Social Science (ICISS 2019), Manila, Philippines (pp. 12-14).

Zhou, Q. (2019). Understanding User Behaviors of Creative Practice on Short Video Sharing Platforms - A Case Study of TikTok and Bilibili (Electronic Thesis or Dissertation).

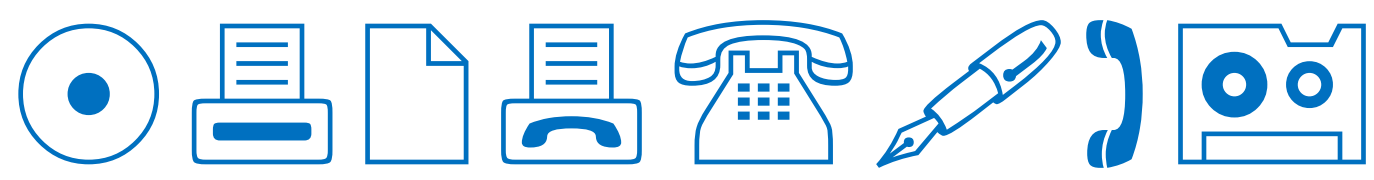

